\title{
Line bisection performance in right-handed primary headache sufferers
}

\author{
Xingyue Hu, Yuhong Liu*, Xinmin Liu**, Mowei Shen***, Roger A. Drake ${ }^{* * * *}$, Wei Wang* \\ Department of Neurology, Sir Run Run Shaw Hospital, Zhejiang University, Hangzhou, *Department of Clinical Psychology and \\ Psychiatry, Zhejiang University, Hangzhou, **Department of Psychology, Southern Anhui Medical College, Wuhu, ${ }^{* *}$ Department of Psychology \\ and Behavioral Sciences, Zhejiang University, Hangzhou, China; ${ }^{* * * * D e p a r t m e n t ~ o f ~ P s y c h o l o g y, ~ W e s t e r n ~ S t a t e ~ C o l l e g e ~ o f ~ C o l o r a d o, ~}$ \\ Gunnison, Colorado, USA
}

\begin{abstract}
Context: In previous studies, patients with migraine and tension-type headaches have shown asymmetries at the central nervous system level. Aims: Hence we would like to figure out whether the lateral cerebral dominance might be more pronounced in the line bisection performance with these patients. Settings and Design: Patients were enrolled in a specialized headache clinic and healthy volunteers from a community as controls. Materials and Methods: The visual line bisection is used to test the unilateral neglect of subjects. Altogether, we studied 28 patients with chronic tension-type, 16 frequent episodic tension-type headache, 31 migraine patients without aura between attacks and 146 healthy volunteers. Statistical Analysis: One-way ANOVA was applied to the mean Index and Net of line bisection errors and the Spearman rank order to the relationship between the Index, Net, subject's age and time since onset of head pain. Results and Conclusions: As reflected by group means of Index of line bisection errors, healthy subjects and migraine patients bisected slightly rightward. Conversely, both forms of tension-type headache sufferers bisected significantly leftward compared to the healthy subjects as well as the migraine sufferers. The study indicates relatively strong right or weak left hemisphere activation or both in the two forms of tension-type headaches, confirming the central nervous system alterations in such patients.
\end{abstract}

Key words: Cognition, line bisection, migraine, neuropsychology, tension-type headache

Migraine and tension-type headaches are two common forms of primary headache. Migraine is characterized by a unilateral head pain location, pulsating quality, moderate or severe intensity, aggravation by routine physical activity and association with nausea, photophobia or phonophobia. Tension-type headache is characterized by a whole scalp pressing or tightening pain, mild to moderate intensity, but not aggravation by routine physical activity and seldom associated with nausea, photophobia or phonophobia. ${ }^{[1]}$ Although the exact mechanisms of these primary headaches are still not fully understood at present, several cerebral or brainstem structures might be involved..$^{[2-4]}$ In addition, a functional cerebral asymmetry in these headaches has been reported. For instance, despite the controversies of EEG data obtained in migraine, a group of investigators ${ }^{[5]}$ enrolled 22 patients with migraine without aura, 20 with migraine with aura and 20 age-matched controls and did find an increased left occipital EEG activity during and between migraine attacks, which might denote a hyperactive left hemisphere in this headache type. The absolute right-left amplitude ratio of patternvisual evoked potentials was higher in migraine between attacks. ${ }^{[6]}$ The lateralized pain in relation to the lateralized brainstem activation after glyceryl trinitrate in migraine ${ }^{[7]}$ and a hypoactivity of the orbitofrontal region in the analgesic-overuse migraine ${ }^{[8]}$ have also been demonstrated using PET imaging. Moreover, pupillary sympathetic hypofunction and asymmetry were reported in both chronic tension-type headache and migraine. ${ }^{[9]}$

Whether these asymmetries are due to the pain experienced remains unknown. Some physiological studies do show that the right hemisphere is more activated than the left by painful stimuli. ${ }^{[10]}$ In a somatosensory evoked potential study, the earlier components were higher when the stimulation was applied to the ipsilateral side of the facial pain. ${ }^{[11]}$ In right-handed patients with complex regional pain syndrome, cortical representation of the pain-affected hand was significantly smaller than that of the contralateral healthy hand. ${ }^{[12]}$

In healthy subjects, the right hemisphere plays a special role in processing the cognitive components of 
social-emotional information, in attention, reasoning and decision-making. The left hemisphere, on the other hand, is involved in aggression, risk-taking and inhibiting the right hemisphere through the corpus callosum, an effect that increases with age. ${ }^{[13]}$ The functional asymmetry of the cerebral hemispheres has been clinically demonstrated in patients with right hemispheric lesions who fail to attend, report or respond to stimuli (visuospatial neglect) in one hemispace and which cannot be attributed to primary sensory or motor deficits. ${ }^{[14]}$

One very simple clinical test of unilateral neglect is the visual line bisection. ${ }^{[15]} \mathrm{A}$ subject is presented with a set of horizontal lines and asked to bisect each line by making a mark in the middle. Lateral deviations from the true center indicate relative inattention for the contralateral side of space. In healthy subjects of the Western world, a leftward error indicating a relatively more active right hemisphere, has been consistently reported; $;^{[16]}$ this is in contrast with the rightward error found in the Eastern culture such as in Japan. ${ }^{[17]}$ Clinical research suggests that patients with unilateral lesions consistently make errors that are directionally specific. For example, errors to the right are more pronounced in patients suffering from a lesion of the right hemisphere, especially involving the inferior parietal and temporoparietal regions. ${ }^{[18,19]}$

Although at present, there have been no reports showing hemispherical lesions in migraine nor in tension-type headache, the high aggression found in migraine ${ }^{[20]}$ and high submissiveness (which is comparable to low aggression) in frequent episodic tension-type headache ${ }^{[21]}$ might imply cerebral asymmetries in these headache types. We therefore hypothesized that migraine sufferers would bisect lines more rightward than healthy subjects and patients with tension-type headaches. Considering that there is no correlation between the side of the unilateral head pain and the dominant hand ${ }^{[22]}$ and that in Chinese culture, most people are trained to use their right hands as artful ones in their lives, ${ }^{[23]}$ while such training in athletes results in consistently rightward errors in line bisection task, ${ }^{[24,25]}$ we selected moderate to strong right-handed subjects in the present study.

\section{Materials and Methods}

\section{Subjects}

This study consisted of 221 Chinese subjects. The healthy volunteers $(n=146)$ were recruited from students, hospital staff or paid volunteers. After a semistructured clinical interview, they were ascertained not to be suffering from any kinds of recurrent headaches. The outpatients were recruited from a headache clinic, diagnosed as suffering from either chronic tension-type headache ( $\mathrm{CTH}, \mathrm{n}=28$ ), frequent episodic tension-type headache (FETH, $\mathrm{n}=16$ ) or migraine without aura ( $\mathrm{n}$ $=31$ ), according to the classification criteria of the International Headache Society. ${ }^{[1]}$ Subjects' age and gender distributions are summarized in Table 1. There were more migraine women than men enrolled in this study, which was in compliance with the epidemiological findings. ${ }^{[26]}$ Patients suffering from mixed migraine plus tension-type headaches were excluded from the study. Migraine subjects were studied between attacks and most CTH or FETH subjects complained of head pains in the whole scalp. The time since onset of head pain varied between three to 360 months in patients and those for the migraine headache were over 6 months. About $50 \%$ of patients had received their headache prophylactic therapies before, but no participants had ingested alcohol, drugs or medication for at least $72 \mathrm{~h}$ prior to the test. No significant group difference by age (one-way ANOVA, main effect, $\mathrm{F}(3,217)=1.56$, $\mathrm{P}$ $=0.20$ ) was detected. This study protocol was approved by the local ethics committee and all subjects gave their written informed consent.

Handedness was determined using a Chinese translation of the Edinburgh Handedness Inventory. ${ }^{[27]}$ Such an inventory has been used in a Chinese study before. ${ }^{[28]}$ Each of the 12 items of the inventory was scored 1, 2 or 3 according to the left-hand, either left or right or right preference. All subjects scored between 29 and 36 and were considered to be moderate or strong right-handers. Their vision was either normal or corrected to normal.

\section{Procedures}

All subjects were requested to bisect eight lines without measuring or folding the paper. The lines, drawn in black and oriented horizontally, ranged from $102-144 \mathrm{~mm}$ in length, were arranged randomly on a sheet of A4 size paper one below the other, differing in their distances from the sheet margins so that their centers were not in alignment. The response sheet was always centered on the subject's mid-sagittal plane. No restrictions were placed on head or eye movements and no time limits were imposed. Subjects were instructed to use their right hand to make a mark indicating the center of the line.

\section{Data analyses and statistics}

There are many classical methods to analyze the line

\begin{tabular}{lccc}
\hline \multicolumn{2}{c}{$\begin{array}{c}\text { Table 1: Age (in years) and gender distribution in healthy } \\
\text { subjects, chronic type headache (CTH), frequent episodic } \\
\text { tension-type headache (FETH) and migraine sufferers }\end{array}$} \\
\hline \multirow{4}{*}{ Mealthy subjects } & $29.0 \pm 8.9$ & $17-47$ & $72 \mathrm{f}, 74 \mathrm{~m}$ \\
CTH & $27.7 \pm 7.3$ & $17-41$ & $9 \mathrm{f}, 19 \mathrm{~m}$ \\
FETH & $30.7 \pm 9.1$ & $19-45$ & $4 \mathrm{f}, 12 \mathrm{~m}$ \\
Migraine & $32.1 \pm 8.6$ & $17-48$ & $26 \mathrm{f}, 5 \mathrm{~m}$ \\
\hline
\end{tabular}

Note: Repeated ANOVA did not detect any statistically significant differences of age and gender distributions between groups; see text for detail 
bisection performance, for instance the percentage expression of bias errors. ${ }^{[29]}$ Here we used a method developed by Drake and Ulrich. ${ }^{[30]}$ Briefly, the distance of the line bisecting mark was measured from the actual center to the nearest millimeter. The frequency of the directional errors, irrespective of the magnitude, was measured using the Index of the Line Bisection Error (Index). This was calculated as (Right - Left)/ (Right + Left); positive values indicate errors to the right and negative ones indicate errors to the left. The magnitude of line bisection deviation was calculated as the algebraic sum of the distance of marks from the veridical center. The statistic is called the Net of Bisection Errors (Net). Positive values indicate errors to the right and negative ones indicate errors to the left.

The mean Index and Net data in the four groups were submitted to the repeated-measurement ANOVA followed by Duncan's multiple new range test. The relationship between the Index, Net, subject's age and time since onset of head pain was assessed by the Spearman rank order correlation test. When referring to the group difference at an individual parameter, Cohen's d (effect size) was also calculated as a supplement indicator for the variation magnitude. With the present sample size, power to detect an effect was larger than $81.3 \%$ (one variable) at $\mathrm{P}<0.05$ in a sample of 16 subjects per group (the smallest group in the present study).

\section{Results}

On an average, healthy subjects and migraine sufferers bisected slightly rightward, whereas patients with CTH and FETH bisected slightly leftward. The individual Index data were widely scattered in each group [Figure 1]. When referring to Index, ANOVA detected statistical significance among the four groups (main effect, $\mathrm{F}$ [3,

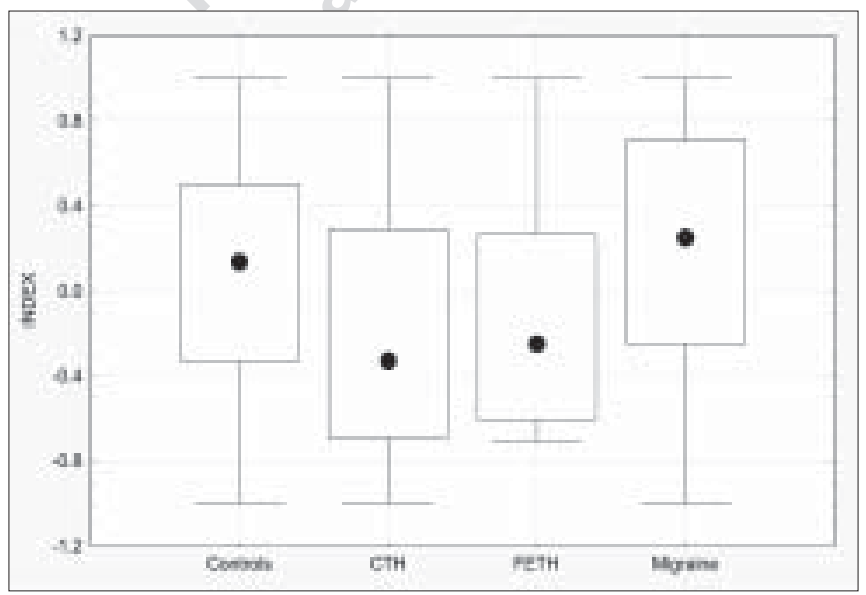

Figure 1: Box and Whisker plot of individual Index of line bisection errors in healthy subjects (controls, $n=146$ ), chronic tension-type headache (CTH, $n=28)$, frequent episodic tension-type headache (FETH, $n=16)$ and migraine $(n=31)$ sufferers. Bars: minimum and maximum values; filled circle: median value; box: quartile. Positive value indicates the rightward bisection error, negative leftward
$217]=4.39, P=0.005)$. The differences between groups were confirmed by Duncan's test. Gender effect was not significant on the mean values of Index between groups $(\mathrm{F}[3,217]=.67, \mathrm{P}=0.41)$. Compared to the healthy controls (mean Index, $0.11 \pm 0.50$ ), both CTH $(-0.19 \pm 0.62$, Cohen's $d=-0.60$ vs. healthy controls $)$ and FETH $(-0.17 \pm 0.50, d=-0.56)$ groups bisected significantly leftward. Although the migraine group bisected more rightward, their mean Index $(0.22 \pm 0.62$, $\mathrm{d}=0.22$ ) was not significantly different from that of the healthy controls. Both group means of Index of CTH (d $=-0.66$ vs. migraine) and FETH $(d=-0.64)$ were also significantly different from that of migraine. Although there were trends for the rightward preference of Net in both CTH (Cohen's $d=-0.44$ vs. the healthy controls) and FETH ( $d=-0.41)$ compared to the healthy controls or migraine, such differences did not reach the level of statistical significance (main effect, $\mathrm{F}[3,217]=1.63$, $P=0.18$ ) [Figure 2].

Since patients with migraine were in the interictal phase and most tension-type headache sufferers localized head pains in the whole scalp, we did not study the line bisection errors in respect to the right or left scalp localization of pain. In 75 patients, there was no significant correlation between the subject's age and Index ( $\mathrm{r}=0.09, \mathrm{P}=0.19)$ or Net $(\mathrm{r}=0.04, \mathrm{P}=0.54)$, nor between the time since onset of head pain and Index ( $\mathrm{r}$ $=-0.06, P=0.37)$ or $\operatorname{Net}(r=0.00, P=0.96)$, nor between headache frequency and Index $(\mathrm{r}=-0.09, \mathrm{P}=0.18)$ or Net $(r=-0.02, P=0.79)$, nor between headache pain severity and Index $(r=-0.09, r=0.18)$ or $\operatorname{Net}(r=-0.05$, $\mathrm{P}=0.40)$.

\section{Discussion}

In compliance with our prediction, migraine sufferers

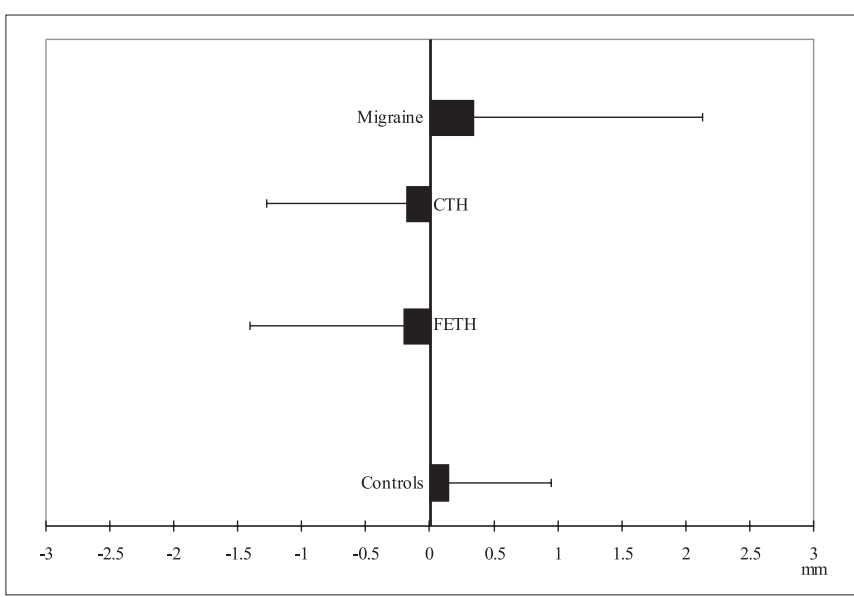

Figure 2: Net of line bisection errors in healthy subjects (controls, $n=146)$, chronic tension-type headache (CTH, $n=28$ ),

frequent episodic tension-type headache $(F E T H, n=16)$ and migraine $(n=31)$ sufferers. Square: mean; Error Bar: S.D. Positive value indicates the rightward bisection error, negative leftward 
bisected lines slightly rightward, which were similar to the healthy subjects. Both CTH and FETH patients however, bisected significantly leftward compared to the healthy subjects. The slight rightward errors found in healthy subjects were in contrast to many documentations of Western countries, ${ }^{[16]}$ but similar to a Japanese study ${ }^{[17]}$ and a Chinese one. ${ }^{[28]}$ The performance discrepancy might result from the fact that our subjects only used their right hand to bisect the lines or from a cultural background that some Chinese people have been forced to switch hand preference from left to the right during their childhood. ${ }^{[23]}$ Another reason could be the gender effect, for example. compared to the healthy men, normal women bisected the lines more leftward ${ }^{[29]}$ and the bias errors were influenced by the menstrual state. ${ }^{[31]}$ Such an influence might be particularly the case in our migraine group which was mainly composed of women subjects. The menstrual fluctuations might affect the hemispheric activation and resulted in non-significant rightward errors in migraine sufferers when compared to those of the healthy subjects. On the other hand, recent results pointed out a cortical hyperexcitability of migraine during the ictal period and to a lesser extent, during the interictal period, ${ }^{[32]}$ which might also contribute to the non-significant findings in our migraine patients.

Some clinical investigations frequently show that tension-type headache sufferers displayed more anxiousness, depression or both compared to patients with migraine or cluster headaches. ${ }^{[33]}$ Such psychological dysregulations accord with the conclusion that the right hemisphere is responsible for stress control. ${ }^{[13]}$ Moreover, stress-induced neurochemical changes may lead to structural and functional alterations in the right hemisphere in psychiatric patients. ${ }^{[34]}$ All these studies were in line with the current findings of the significant leftward bisection errors in both forms of tension-type headaches, which might point to a relative hyperactive right or hypoactive left hemisphere or both.

The performances of line bisection in CTH and FETH groups were similar, the smaller sample size of FETH, i.e., half the size of CTH, might have contributed to the outcome. Nonetheless, this study does not differentiate neuropsychologically between the two headache forms. Neither does it fit into Jensen's ${ }^{[3]}$ assumption that peripheral changes like myofascial nociception are important in episodic tension-type headache while central mechanisms like central sensitization are preponderant in CTH. Indeed, an experimental study of myofascial pain helps to explain the increased pain response in episodic tension-type headache patients; the sensitization of peripheral nociceptors per se were experienced through central mechanisms. ${ }^{[35]}$

Whether our findings were related to the head pain experienced still remains unclear. In other patients suffering from complex regional pain syndrome, line bisection errors were not significantly different from errors of the healthy subjects. ${ }^{[36]}$ There was, however, a suggestion that the right frontal brain hyperactivity (reflected by the central zone or parietal zone EEG asymmetry) was correlated with enhanced pain sensitivity and negative affect. ${ }^{[37]}$ Furthermore, the pressure pain detection and tolerance thresholds to mechanical stimuli were decreased in tension-type headache sufferers. ${ }^{[38,39]}$

One obvious limitation of our study is that all migraine sufferers were without aura and were studied in their interictal period. Visual aura might deteriorate the cognitive functions, as reflected by a visual evoked potential study. ${ }^{[40]}$ Although some investigations might indicate the possible cognitive dysfunctions in migraine during the interictal period, ${ }^{[41]}$ further investigation on the link between the ictal pain and the different hemispheric activation in migraine is still needed. Other limitations might include the situational factors which were less controlled during the test, the prophylactic therapy which half of our patients received three days before the test and the sample size of FETH which was small. Moreover, our study is a clinically based one and it lacks a comparison between patients and healthy controls regarding the educational experiences received.

\section{Conclusion}

The leftward line bisection errors in both forms of tension-type headaches might indicate a relatively strong right hemispheric activation, a relatively weak left hemispheric activation or both. This finding supports the role of a different pattern of cortical activation in tension-type headaches than that found in healthy controls, but leaves the question open whether the line bisection performance is normal in migraine, both with and without aura, during the ictal phase.

\section{Acknowledgments}

The correspondent author was partly supported by the Department of Science and Technology of Zhejiang Province (No. 2005C33022), the Young Investigator's Fund of the Department of Health of Zhejiang Province (No. 2005QN011), the Natural Science Fund of Zhejiang Province, (No. X206965), the 151 Intelligence Fund of the Department of Personnel of Zhejiang Province and the Research Center of Language and Cognition of Zhejiang University. Drs. Xingyue Hu and Yuhong Liu contributed equally to the paper.

\section{References}

1. Headache Classification Committee of the I nternational $\mathrm{H}$ eadache Society. The International Classification of headache disorders, $2^{\text {nd }}$ ed.

2. Schoenen J, B endtsen L. N europhysiology of tension-type headache. In: Olesen J , T felt-H ansen P, Welch K M , editors. The headaches. L ippincott Williams and Wilkins: P hiladelphia; 2000. p. 579-87. 
3. J ensen R. Peripheral and central mechanisms in tension-type headache: An update. Cephalalgia 2003;23:49-52.

4. May A. The contribution of functional neuroimaging to primary headaches. N eurol Sci 2004;25:S85-8.

5. N eufeld MY, Treves TA, Korczyn AD. E E G and topographic frequency analysis in common and classic migraine. H eadache 1991;31:232-6.

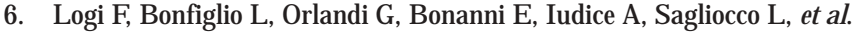
Asymmetric scalp distribution of pattern visual evoked potentials during interictal phases of migraine. Acta N eurol Scand 2001;104:301-7.

7. Afridi SK, Matharu MS, L ee L, K aube H, Friston KJ , Frackowiak RS, \& al. A PET study exploring the laterality of brainstem activation in migraine using glyceryl trinitrate. Brain 2005;128:932-9.

8. Fumal A, L aureys S, Clemente Di, B oly L, B ohotin M, Vandenheede V, $A$ al. Orbitofrontal cortex involvement in chronic analgesic-overuse headache evolving from episodic migraine. B rain 2006;129:543-50.

9. Takeshima T, Takao Y, Takahashi K. Pupillary sympathetic hypofusion and asymmetry in muscle contraction headache and migraine. Cephalalgia 1987;7:257-62.

10. Otto MW, Dougher MJ, Yeo RA. Depression, pain and hemispheric activation. J Nerv Ment Dis 1989;177:210-8.

11. Tinazzi $M$, Valeriani $M, M$ oretto $G, R$ osso T, N icolato A, F iaschi A, etal. Plastic interactions between hand and face cortical representations in patients with trigeminal neuralgia: A somatosensory-evoked potentials study. N euroscience 2004;127:769-76.

12. Pleger $B$, Tegenthoff $M$, Schwenkreis $P, J$ anssen $F, R$ agert $P, D$ inse $H R$, đ al. M ean sustained pain levels are linked to hemispherical side-to-side differences of primary somatosensory cortex in the complex regional pain syndrome I. Exp B rain Res 2004;155:115-9.

13. Schore AN. E ffects of a secure attachment relationship on right brain development, affect regulation and infant mental health. Infant Ment H ealth J 2001;22:7-66.

14. Roberts IH H alligan PW. Spatial neglect: A clinical handbook for diagnosis and treatment. E rlbaum: H ove, UK; 1999.

15. H eilman K M, van den Abell T. Right hemisphere dominance for attention: The mechanism underlying hemispheric asymmetries for inattention (neglect). N eurology 1980;30:327-30.

16. J ewell G, McCourt ME. Pseudoneglect: A review and meta-analysis of performance factors in line bisection tasks. N europsychologia 2000;38:93110.

17. Fujii T, Fukatsu R, Yamadori A, Kimura I. Effects of age on the line bisection test. J Clin Exp N europsychol 1995;17:941-4.

18. Schenkenberg T, B radford DC, Ajax ET. Line bisection and unilateral visual neglect in patients with neurological impairment. N eurology 1980;30:509-17.

19. M ennemeier M, Vezey E, L amar M, J ewell G. Crossover is not a consequence of neglect: A test of the orientation/estimation hypothesis. J Int N europsychol Soc 2002;8:107-14.

20. Cao M, Zhang S, Wang K, Wang Y, Wang W. Personality traits in migraine and tension-type headaches: A five-factor model study. Psychopathology 2002;35:254-8.

21. Wang W, Yang T, Zhu H, Mao F, Livesley WJ, Larstone RM, \& al. Disordered personality traits in primary headaches. Soc B ehav Person
2005;33:495-501

22. L ipscombe SL, Prior T. Is there any relationship between handedness and unilateral headache in migraine? Cephalalgia 2002;22:146-8.

23. H oosain R. L eft handedness and handedness switch amongst the Chinese. Cortex 1990;26:451-4.

24. Carlstedt RA. L ine-bisecting performance in highly skilled athletes: D oes preponderance of rightward error reflect unique cortical organization and functioning? B rain Cogn 2004;54:52-7.

25. Dumbrava A, Tatu M K. Visuo-spatial pseudohemineglect in professional sportsmen. Cogn Process 2006;7:124

26. Rasmussen BK. E pidemiology of headache. Cephalalgia 1995;15:45-68.

27. Oldfield RC. The assessment and analysis of handedness: The E dinburgh I nventory. N europsychologia 1971;9:97-113.

28. Wang W, Wang Y, Gu J , Drake RA, Livesley WJ , J ang K L. L ine bisection performance in patients with personality disorders. Cognit N europsychiatry 2003;8:273-85.

29. H ausmann M, E rgun G, Yazgan Y, Gunturkun O. Sex differences in line bisection as a function of hand. N europsychologia 2002;40:235-40.

30. Drake RA, U Irich $G$. L ine bisecting as a predictor of personal optimism and desirability of risky behaviors. Acta Psychol (Amst) 1992;79:219-26.

31. H ausmann M. H emispheric asymmetry in spatial attention across the menstrual cycle. N europsychologia 2005;43:1559-67.

32. Welch K M. Contemporary concepts of migraine pathogenesis. N eurology 2003;61:S2-8.

33. B ertolotti G, Vidotto G, Sanavio E, Frediani F. Psychological and emotional aspects and pain. N eurol Sci 2003;24:S71-5.

34. Cutting $\mathrm{J}$. The role of right hemisphere dysfunction in psychiatric disorders. B r J Psychiatry 1992;160:583-8.

35. Mork $H, A$ shnia $M, B$ endsten $L$, Olesen J , J ensen R. Possiblemechanisms of pain perception in patients with episodic tension-type headache. A new experimental model of myofascial pain. Cephalalgia 2004;24:466-75.

36. Forderreuther $S$, Sailer $U$, Straube A. Impaired self-perception of the hand in complex regional pain syndrome (CRPS). Pain 2004;110:756-61.

37. Pauli P, Wiedemann G, Nickola M. Pain sensitivity, cerebral laterality and negative affect. Pain 1999;80:359-64.

38. Schoenen J, Bottin D, H ardy F, Gerard P. Cephalic and extracephalic pressure pain thresholds in chronic tension-type headache. Pain 1991;47:145-9.

39. B endtsen $L$, J ensen R, Olesen J . Qualitatively altered nociception in chronic myofascial pain. Pain 1996;65:259-64.

40. K halil N M, L egg NJ , Anderson DJ . L ong term decline P 100 amplitude in migraine with aura. J N eurol N eurosurg Psychiatry 2000;69:507-11.

41. A mbrosini A, de N oordhout AM, Sandor PS, Schoenen J . E lectrophysiological studies in migraine: A comprehensive review of their interest and limitations. Cephalalgia 2003;23:13-31.

\section{Accepted on 23-06-2007}

Source of Support: Zhejiang Province and the Research Center of Language and Cognition of Zhejiang University,

Conflict of Interest: None declared. 